\title{
Ultrasound guided percutaneous radiofrequency thermal ablation of symptomatic uterine fibroids - results from a single center and 52 weeks of follow up
}

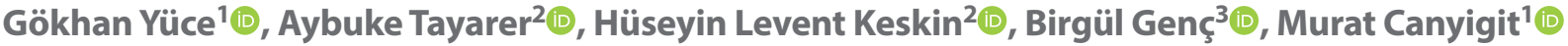 \\ ${ }^{1}$ Ankara City Hospital, Interventional Radiology Department, Turkey \\ ${ }^{2}$ Ankara Yildirim Beyazit University, Gynecology and Obstetrics Department, Turkey \\ ${ }^{3}$ Ankara City Hospital, Endocrinology Department, Turkey
}

\begin{abstract}
Objectives: Uterine fibroids are one of the most common female disorder of the reproductive age and may cause abnormal uterine bleeding (UAB), pain or infertility. Our aim was to evaluate the safety and efficacy of percutaneous radio frequency ablation (RFA) in reducing clinical symptoms, fibroid volume and improving laboratory parameters.

Material and methods: Thirty-five symptomatic patients with 54 uterine fibroids were enrolled. Preintervention evaluation was made for each participant and included ultrasonography to assess the volume, largest diameter and location of the fibroid and Visual Analogue Scale (VAS) for quantifying the degree of menstrual pain. The magnitude of menstrual bleeding was scored for each patient by using pictogram. Preprocedural laboratory assessment included hemoglobulin and hematocrit. Treatment efficacy was evaluated at 3, 6 and 12 months after the intervention with ultrasound (US) measurements, symptom scores and laboratory parameters.

Results: Pretreatment mean Hb was significantly lower than those at 3, 6 and 12 month post treatment visits $(p<0.001)$. The pretreatment median volume was significantly higher than the median volumes measured at 3,6 and 12 months after RFA $(p<0.001)$. Visual Analogue Score (VAS) for pain was significantly lower than baseline values at 6 and 12 month visits $(p<0.01)$. Pretreatment bleeding scores and the number of patients in the predefined severe bleeding category were significantly decreased.

Conclusions: US guided RF ablation of uterine fibroids is relatively safe and effective procedure. It can be applied to the fibroids with varying localizations and sizes. It reduces the fibroid volume and obviate a need for more invasive treatment. Key words: uterine fibroids; percutaneous; radiofrequency ablation
\end{abstract}

Ginekologia Polska 2020; 91, 8: 447-452

\section{INTRODUCTION}

Uterine fibroids are the most common benign tumors of the female reproductive system and have a significant effect on quality of life, economy and reproduction. Its prevalence was reported as $70 \%$ in Caucasian woman and $80 \%$ in Afro-Americans [1] .They constitute a big proportion of hysterectomy indications. In one report the annual cost of the disease and its obstetric complications was reported as $\$ 5.89$ billion-\$34.37 billion [2].

Uterine fibroids are classified in regard to their location in the uterus as intramural (completely or mostly localized within the myometrium), submucosal (projecting into the endometrial cavity and may be pedunculated), or sub serosal (projecting outward from the serosal surface of uterus and may be pedunculated) [3].They may be asymptomatic and discovered during routine examination [4]. Nevertheless, almost half of the individuals admit with clinical symptoms such as abnormal uterine bleeding (AUB), menstrual or intermenstrual pain, discomfort in pelvis region, infertility and consequent reduced quality of life $[5,6]$. Diagnosis is usually made by ultrasound (US) which enables vaginal exploration and is very accurate and available except large fundal fibroids [7] .

Recent treatment options are medical, surgical or minimally invasive techniques. Surgery is associated with complications and can cause significant morbidity [8]. Moreover, it undesirable to women who are planning future pregnancies. Myomectomy is a less invasive surgical procedure and is 
still associated with perioperative challenges such as bleeding control, adhesions, rare cases of uterine rupture and possible need for conversion of surgery to hysterectomy.

As the technology develops, less invasive interventional techniques have become more popular, which allow the selective destruction of the fibroids. Those are endovascular uterine artery embolization (UAE), high intensity focused ultrasound (HIFU) and radiofrequency ablation (RFA) $[6,9,10]$. Uterine artery embolization (UAE) isn't target selective and isn't recommended if pregnancy is desired. In addition to that, recurrent fibroid may develop and $20 \%$ of patients may require subsequent surgery [10].

RFA is an available option as a treatment modality for fibroids and can be performed via percutaneous or transvaginal route. In this technique, energy is released directly within the fibroid via electrodes without giving damage to the adjacent tissues. RFA reduces the volume of the fibroid and relieves associated symptoms [11, 12].

\section{Objectives}

In this study, we aimed to evaluate the safety and efficacy of percutaneous RFA in relieving clinical symptoms, laboratory parameters and size of the fibroids in 52 weeks of the follow up period.

\section{MATERIAL AND METHODS}

In this prospective study, 35 patients with symptomatic uterine fibroids who admitted to our tertiary center's Gynecology and Obstetrics clinics between November 2017 and October 2018 and required treatment were enrolled. Our local ethics committee approved the study protocol in accordance with principles of the Declaration of Helsinki. The patients recruited in the study were informed about the alternative treatment options including arterial embolization and surgery and the possible advantages and risks of RFA before obtaining the written informed consent. None of them had a comorbidity which could be a contraindication for the intervention, sedation or local anesthesia. The patients who were younger than 18 years of age, had active genitourinary infection, history of coagulation abnormality, present pregnancy/lactation or planning pregnancy were excluded.

Before the procedure all patients were assessed by the same gynecologist and radiologist. The gynecologist performed Visual Analogue Scale (VAS) in order to quantify the pain. The patient was requested to choose a score between 0-10 for dysmenorrhea (0 represented no pain and 10 was maximum pain). The magnitude of menstrual bleeding was also classified for each patient as amenorrhea, mild, moderate or severe bleeding. Pictorial Blood Loss Assessment Chart (PBAC) was used for that purpose and score 0 was defined as amenorrhea, score $0-50$ as mild bleeding, score
50-100 as moderate bleeding and score $>100$ as severe bleeding. Pretreatment evaluation included conventional transabdominal US and transvaginal ultrasound US (Logiq E9, GE Healthcare, Milwaukee, WI), equipped with a 3-5 MHz convex probe and a 1-6 MHz transvaginal probe. Data derived from pretreatment ultrasonography assessment of the fibroids was composed of the number, location (fundal, anterior, posterior, right side, left side, right horn, left horn), situation (subserosal, intramural or submucosal), the largest diameter and volume (volume $=0.5233 \times$ Anteroposterior $x$ Transverse $x$ Longitudinal Dimension). The measurements were repeated 3, 6 and 12 months after RFA. Preprocedural hemoglobulin $(\mathrm{Hb})$ and hematocrit $(\mathrm{Htc})$ were recorded at baseline and reevaluated at 3, 6 and 12 months after RFA.

The RFA procedure was conducted in the interventional radiology department under intravenous (iv) sedation with 1-3 mg of midazolam, 25-50 mcg of fentanyl or 50-120 mg of propofol. During the RFA, patients were in supine position and monitored by means of electrocardiogram (ECG), blood pressure and pulse. Broad-spectrum antibiotic prophylaxis was given an hour before the procedure. A uterine manipulator was applied to allow fixation only in those patients whose fibroids were difficult to puncture due to either their rigid structure or challenging locations. Percutaneous RF was performed after the fibroid had been punctured under abdominal US monitoring by using a coaxial system with a 35 to $40 \mathrm{~mm}$ long umbrella-shaped needle-electrode (Med sphere, RF3000 system, Shanghai, China) (Fig. 1). One or more thermal ablations were performed depending on the size of the fibroid. Maximum intensity was $100 \mathrm{~W}$ during the ablation. The ultrasonographic appendence of the same fibroid before, during and after RFA were demonstrated on Figure $1 \mathrm{a}-\mathrm{d}$.

After RFA, the patient was advised to take paracetamol for one or two days if needed and continue to the antibiotic treatment for one week. All complications that could be

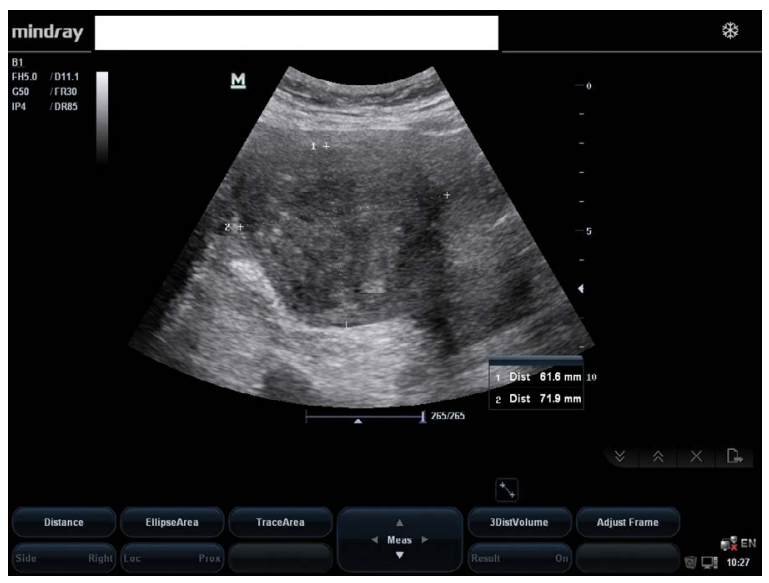

Figure 1a. Ultrasonıgraphic image of the uterine fibroid before RFA 


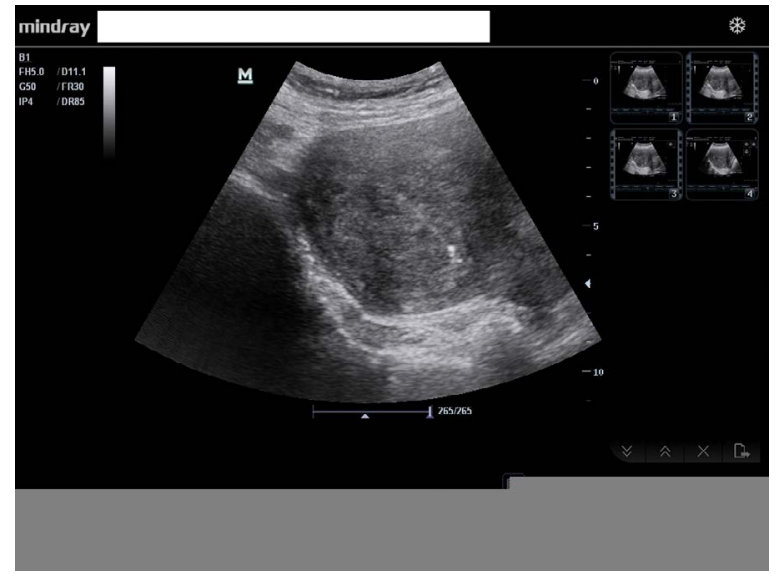

Figure 1b. RF needle placement within the fibroid during RFA

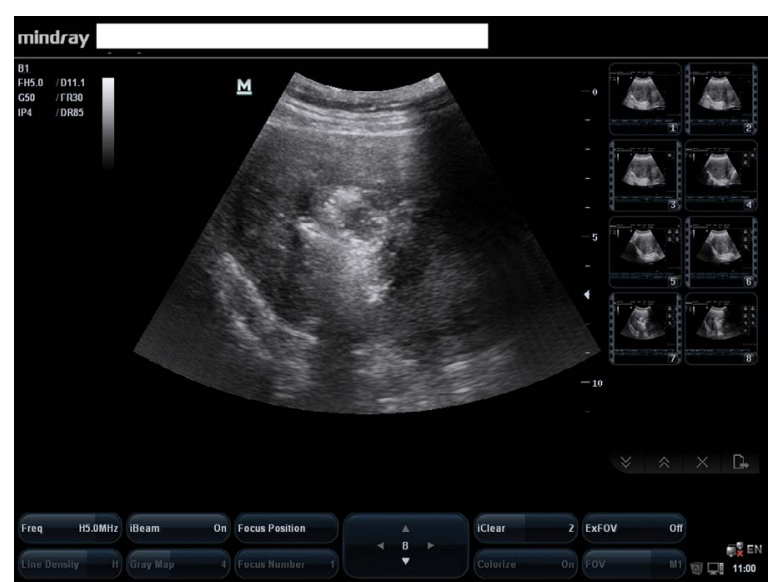

Figure 1c. Myolysis during RFA

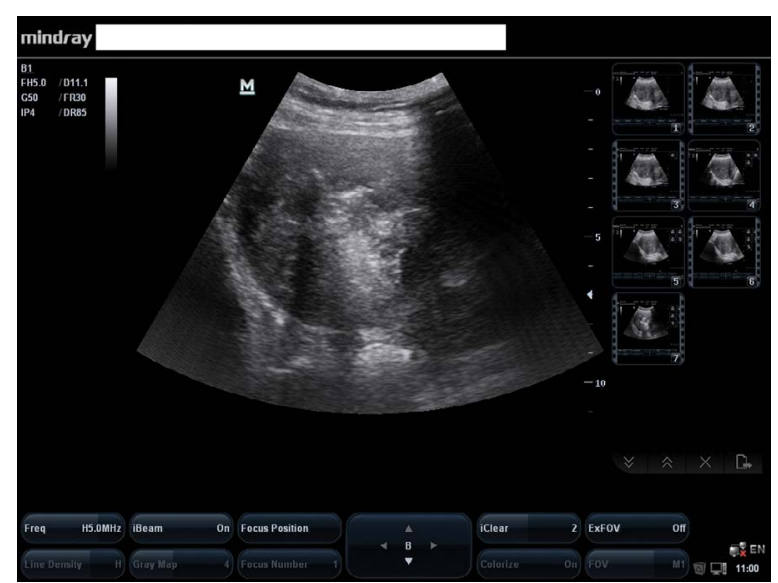

Figure 1d. Ultrasonographic image of the fibroid after RF

associated with RFA were recorded right after and at the first visit 1 week after the intervention. Patients were called for control visits at 3, 6 and 12 months after RFA and US examination, symptom scores and laboratory parameters
Table 1. Baseline characteristics of 35 patients with 54 fibroids underwent RF ablation

\begin{tabular}{|l|l|}
\hline Mean age (years) & $35.36 \pm 7.362$ \\
\hline Nulligravida (number of patients) & 5 \\
\hline Solitary fibroid (number of patients) & 24 \\
\hline More than 1 fibroid (number of patients) & 11 \\
\hline Type of fibroid intramural/subserozal (number) & $(46 / 8)$ \\
\hline $\begin{array}{l}\text { Location of the fibroids ( } \neq \text { of fibroids) } \\
\text { Anterior }\end{array}$ & 16 \\
$\begin{array}{l}\text { Posterior } \\
\text { Fundus } \\
\text { Right Horn }\end{array}$ & 8 \\
\hline
\end{tabular}

$\mathrm{RF}$ - radio frequency

were reassessed. Successful treatment was clinically considered when observing a reduction in symptoms 6 months after RFA. Successful treatment was also defined when the achieved fibroid necrosis at 6 months after treatment was larger than $50 \%$.

\section{Statistics}

The Statistical Package for the Social Sciences, version 25 (SPSS Inc., Chicago, IL, USA) was used for the statistical analysis. Descriptive statistics are presented as means \pm standard deviation (SD) and medians (minimum-maximum) for continuous variables and as percentages (\%) for categorical variables The nonparametric Kolmogorov-Smirnov test was used to compare samples with the reference probability distribution, whereas the homogeneity of the variances assessed using the Levene test. The nonparametric Friedman test was used in order to compare initial and last measured volume and longest axis of the nodules before and after intervention. $\mathrm{P}<0.05$ was accepted as statistically significant.

\section{RESULTS}

Thirty-five symptomatic patients with 54 uterine fibroids who were unresponsive to medical therapy or didn't decide to undergo surgery and gave consent for RF treatment were enrolled in the study. The mean age was $35.36 \pm 7.362$ ( $\min -\max ; 25-47$ ). Five patients were nulligravida, 17 had 2 gestations whereas the remaining had history of $\geq 3$ previous gestations. Twenty-four patients had one fibroid whereas remaining eleven had multiple fibroids. The location of the fibroids was anterior in 16 , posterior in 8 , fundus in 22 and 8 in the right horn. Regarding the type of the fibroid, 46 were intramural whereas 8 were subserosal. The baseline characteristics of the patients were demonstrated on Table 1. The number of ablations performed for each patient was 2.7 (changing between 1-5 based on size). There wasn't any intervention related severe complication 
Table 2. Demonstration and comparison of pre and post ablation $\mathrm{Hb}$, longest diameter, volume, pain and bleeding scores of the patients

\begin{tabular}{|c|c|c|c|c|c|}
\hline & Pre-ablation & Post RF 3 months & Post RF 6 months & Post RF 12 months & $\mathbf{p}^{*}$ \\
\hline $\mathrm{Hb} \mathrm{g} / \mathrm{dL}$ mean $\pm \mathrm{SD}$ & $11.56 \pm 1.65$ & $12.5 \pm 1.42$ & $13.17 \pm 1.33$ & $13.37 \pm 4.47$ & $\mathrm{p}<0.001$ \\
\hline Longest diameter mm median (min-max) & $72(44-124)$ & $56(36-112)$ & $52(26-106)$ & $53(18-120)$ & $\mathrm{p}<0.001$ \\
\hline Volume $\mathrm{mL}$ median (min-max) & $85(17-519)$ & $64(13-330)$ & $50(3.4-372)$ & $57(2.2-518)$ & $p<0.001$ \\
\hline Mean Menstrual Pain VAS & $7.95 \pm 1.35$ & $6.32 \pm 1.25$ & $4.74 \pm 1.32$ & $4.47 \pm 1.12$ & $p<0.001$ \\
\hline \multicolumn{6}{|l|}{ Bleeding severity (number of patients/\%) } \\
\hline $\begin{array}{l}\text { Amenorrhea } \\
\text { Mild } \\
\text { Moderate } \\
\text { Severe }\end{array}$ & $\begin{array}{l}0(0 \%) \\
5(14.3 \%) \\
9(25.7 \%) \\
21(60 \%)\end{array}$ & $\begin{array}{l}0 \\
9(25.7 \%) \\
15(42.8 \%) \\
11(31.5 \%)\end{array}$ & $\begin{array}{l}0 \\
11(31.5 \%) \\
14(40 \%) \\
10(28.5)\end{array}$ & $\begin{array}{l}0 \\
15(42.8 \%) \\
14(40 \%) \\
6(8.2 \%)\end{array}$ & $p<0.001$ \\
\hline
\end{tabular}

reported after the procedure. The most prevalent complication was abdominal pain occurred in all except 2 patients which was mild to moderate and responsive to paracetamol treatment. Mild erythema and skin reaction at the entry site of the needle was observed in 19 patients and resolved in few days spontaneously without any need for topical treatments. Four patients reported urinary tract infection symptoms despite prophylactic antibiotics and were prescribed oral ciprofloxacin. None of the patients experienced leukocytosis, fever, nausea, fatigue or malaise after the procedure. Mild vaginal bleeding occurred in 12 patients that resolved in maximum 6 days. The mean time needed for whole procedure was approximately 45 minutes. Uterine manipulator was required only in five cases. All patients were discharged within the day of ablation and none of them required rehospitalization afterwards. During the 52 weeks of follow-up, none of the patients needed a subsequent surgery or another minimally invasive procedure. All patients attended the visits at 3, 6 and 12 months after RFA. The pretreatment mean $\mathrm{Hb}$ was $11.56 \pm 1.65$ whereas posttreatment mean $\mathrm{Hb}$ at 3,6 and 12 months were $12.5 \pm 1.42,13.17 \pm 1.3$ and $13.37 \pm 1.25$, respectively. Posttreatment mean $\mathrm{Hb}$ values were significantly higher than pretreatment mean $\mathrm{Hb}$ $(p<0.01)$ whereas there wasn't any significant difference between the posttreatment $\mathrm{Hb}$ levels at 3,6 and 12 months (Tab. 2). The pretreatment median longest diameter was significantly higher than posttreatment longest diameters ( $p=0.003, p<0.001$ and $p<0.001$, respectively) (Tab. 2). There wasn't any statistically significant difference between the posttreatment median longest diameters. When intramural and submucosal fibroids were compared, there wasn't any statistically significant difference between the baseline mean longest diameters whereas posttreatment longest diameters were significantly smaller for intramural fibroids $(p<0.001)$ at 3, 6 and 12 months after RFA. The pretreatment median volume of the fibroids was significantly higher than posttreatment median volumes at 3, 6 and 12 months after RFA ( $p=0.007, p<0.01$ and $p<0.01$, respectively) (Tab. 2). The median percentage of volume reduction compared to baseline was $36.8 \%(3-72)$ at 3 months whereas it was $50 \%(2-92)$ and $46.8 \%(1-95)$ at 6 and 12 months after RFA, respectively. When intramural and submucosal fibroids were compared, post treatment mean volumes at 3, 6 and 12 months after RFA were significantly smaller and percentage of volume reduction was significantly higher for intramural fibroids ( $p<0.001$ and $p=0.024$, respectively). Pretreatment mean VAS for menstrual pain was $7.95 \pm 1.35$ and it was significantly higher than mean VAS $(p<0.01)$ at posttreatment 6 and 12 months (Tab. 2). Regarding bleeding severity, the number of patients who were experiencing severe menstrual bleeding was significantly reduced in the post treatment follow up (Tab. 2).

\section{DISCUSSION}

Uterine fibroids are the most common benign pelvic tumors. Previous studies have shown that $35 \%$ of premenopausal women had a previous diagnosis of fibroid tumors and $51 \%$ of undiagnosed premenopausal women had ultrasound evidence of fibroid tumors [1]. The location of the tumor, volume and adjacent organs may alter the clinical symptoms. Current treatment modalities for uterine fibroids are surgery or radiological interventions which include UAE or RFA. A hysterectomy can completely cure the uterine fibroids but is considered too radical by most of the patients and fertility is a concern. A myomectomy is less radical, but it can be associated with increased intraoperative bleeding, postoperative infection, pelvic adhesions and omission of tumors, which limits its usage. UAE is a frequently used technique which reduces length of hospital stay and pain compared to the operation and patients rapidly return to the daily usual activities. However, UAE has the risk of reintervention, and lower pregnancy and live birth rates [13].

$\mathrm{RF}$ ablation is used in the treatment of uterine fibroids and has several advantages such as low cost and obviating need for hospitalization. Only one applicator is necessary for a single patient and RF generators are widely available in the interventional radiology units for ablation of wide variety of tumors. Radiofrequency ablation can be performed via 
several routes such as during laparoscopy, percutaneous or transvaginal. In the laparoscopic approach general anesthesia is required which lengthens the hospital stay [14]. However transabdominal percutaneous RF can be applied under sedation and allows rapid recovery and early restoration of the daily activities. In a previous report, percutaneous RF ablation with ultrasound guidance was found to be feasible in symptomatic patients with fibroids $4-6 \mathrm{~cm}$ in diameter [6]. In another study women with symptomatic single or multiple fibroids underwent percutaneous RF and followed for six months. RFA was successful regarding to decreasing fibroid volume, relieving symptoms and increasing quality of life [11]. Those two previously mentioned studies were conducted with limited number of patients ( 9 and 11) and follow up interval was short. In another study with women with large subserosal or intramural fibroids $(>5 \mathrm{~cm})$, the combined treatment with RF following UAE improved symptom severity scores significantly and mean volume reduction was 56.5\% [15]. In our study, the number of patients included was relatively higher than the previous reports and the size and number of the fibroids were varying in a larger range $(4.4-12 \mathrm{~cm})$. In previous reports, it was postulated that RFA was more effective in volume reduction in intramural fibroids compared to submucosal ones [6]. In accordance with those data, the post treatment mean longest diameter and volume of the fibroids were smaller in intramural fibroids compared to submucosal ones and volumetric response to RFA was better in intramural fibroids in our study. However, the number of patients with submucosal polyps was small and not enough to draw a definitive conclusion. RFA was found to be safe with mild to moderate complications including abdominal pain and skin erythema on the entry site of the needle or urinary tract infection easily managed with oral antibiotics and mild vaginal bleeding. RFA significantly reduced the fibroid size and volume and none of the patients required subsequent treatments in one year of follow up. The fibroid size and volume were significantly lower than basal measurements but there wasn't any significant difference between post RF 3, 6 and 12 month measurements. The volume reduction was most prominent on the $6^{\text {th }}$ month visit and median percentage of reduction was $50 \%$ (min-max; 2-92). This reduction is slightly inferior to that shown by Bergamini et al. (77\%) and Ghezzi et al. [16, 17] $(68.8 \%)$ which may be related with the larger sizes of the fibroids in our study compared to previous ones and usage of US as the only imaging modality rather than more precise and less subjective ones such as MRI. There are studies in the literature using different imaging modalities such as contrast enhanced US or MRI $[18,19]$. An MRI is particularly useful to define the exact location of fibroids, being highly accurate in problematic cases, such as large (> $375 \mathrm{~mL})$, fundal or multiple (> 4) fibroids [20]. In the present study, menstrual bleeding score decreased significantly. According to self-assessment scores made before and after ablation, the number of patients who were experiencing severe menstrual bleeding decreased significantly. That result was also reflected as increased $\mathrm{Hb}$ and $\mathrm{Htc}$ levels in the post ablation follow ups. VAS for menstrual pain was also reduced which would increase the quality of life.

In our study, the fibroids were in various locations and some were difficult to reach and manipulate. Since the radiologist was experienced and skilled, those challenging locations didn't constitute an obstacle for RFA. In order to gain experience, it is advised to choose initial cases with one to three fundal myoma [21].

The limitation of our study was the limited number of patients. We need further studies with larger number of patients and with longer follow up to ensure that the volume reduction effect is durable and recurrent symptoms don't reoccur by time. There is also need for further studies to provide information about the possible effects on endometrium or future gestations.

In conclusion, US guided percutaneous RFA of uterine fibroids is relatively safe and effective procedure. It can be applied to the fibroids with varying localizations and sizes. It effectively reduces volume and obviate a need for more invasive treatment. Besides size reduction, it also ameliorates the symptoms such as pain and heavy bleeding which are reducing the quality of life.

\section{Conflict of interest}

The authors of this article confirm that there is no conflict of interest between authors.

\section{Acknowledgements}

All of us have made a substantial contribution to the information submitted and have read the final manuscript. We have no direct or indirect commercial, financial incentive associated with publishing this article. Each of us acknowledges that he or she participated sufficiently in the work to take public responsibility for its content. We thank the staff of interventional radiology department for assisting us during ablation procedures and help as recording all needed data

\section{REFERENCES}

1. Baird DD, Dunson DB, Hill MC, et al. High cumulative incidence of uterine leiomyoma in black and white women: ultrasound evidence. Am J Obstet Gynecol. 2003; 188(1): 100-107, doi: 10.1067/mob.2003.99, indexed in Pubmed: 12548202.

2. Cardozo ER, Clark AD, Banks NK, et al. The estimated annual cost of uterine leiomyomata in the United States. Am J Obstet Gynecol. 2012; 206(3): 211.e1-211.e9, doi: 10.1016/j.ajog.2011.12.002, indexed in Pubmed: 22244472

3. lerardi AM, Savasi V, Angileri SA, et al. Percutaneous High Frequency Microwave Ablation of Uterine Fibroids: Systematic Review. Biomed Res Int. 2018; 2018: 2360107, doi: 10.1155/2018/2360107, indexed in Pubmed: 29511672. 
4. $\mathrm{Kim} \mathrm{CH}$, Kim SR, Lee HA, et al. Transvaginal ultrasound-guided radiofrequency myolysis for uterine myomas. Hum Reprod. 2011; 26(3): 559-563, doi: 10.1093/humrep/deq366, indexed in Pubmed: 21216788.

5. Bulun SE. Uterine fibroids. N Engl J Med. 2013; 369(14): 1344-1355, doi: 10.1056/NEJMra1209993, indexed in Pubmed: 24088094.

6. Jones $\mathrm{S}, \mathrm{O}$ 'Donovan $\mathrm{P}$, Toub $\mathrm{D}$. Radiofrequency ablation for treatment of symptomatic uterine fibroids. Obstet Gynecol Int. 2012; 2012: 194839 , doi: 10.1155/2012/194839, indexed in Pubmed: 21961009.

7. Woźniak A, Woźniak S. Ultrasonography of uterine leiomyomas. Prz Menopauzalny. 2017; 16(4): 113-117, doi: 10.5114/pm.2017.72754, indexed in Pubmed: 29483851.

8. Guarnaccia MM, Rein MS. Traditional surgical approaches to uterine fibroids: abdominal myomectomy and hysterectomy. Clin Obstet Gynecol. 2001; 44(2): 385-400, doi: 10.1097/00003081-200106000-00024, indexed in Pubmed: 11345000.

9. Recaldini $C$, Carrafiello $G$, Laganà $D$, et al. Percutaneous sonographically guided radiofrequency ablation of medium-sized fibroids: feasibility study. AJR Am J Roentgenol. 2007; 189(6): 1303-1306, doi: 10.2214/AJR.07.2184, indexed in Pubmed: 18029862.

10. Kroon B, Johnson N, Chapman M, et al. Australasian CREI Consensus Expert Panel on Trial evidence (ACCEPT) group. Fibroids in infertility-consensus statement from ACCEPT (Australasian CREI Consensus Expert Panel on Trial evidence). Aust N Z J Obstet Gynaecol. 2011; 51(4): 289-295, doi: 10.1111/j.1479-828X.2011.01300.x, indexed in Pubmed: 21806566.

11. Carrafiello G, Recaldini C, Fontana F, et al. Ultrasound-guided radiofrequency thermal ablation of uterine fibroids: medium-term follow-up. Cardiovasc Intervent Radiol. 2010; 33(1): 113-119, doi: 10.1007/s00270009-9707-3, indexed in Pubmed: 19777299.

12. Lee BB, Yu SP. Radiofrequency Ablation of Uterine Fibroids: a Review. Curr Obstet Gynecol Rep. 2016; 5(4): 318-324, doi: 10.1007/s13669-0160183-x, indexed in Pubmed: 27917310.

13. Ludwig PE, HuffTJ, Shanahan MM, et al. Pregnancy success and outcomes after uterine fibroid embolization: updated review of published literature. Br J Radiol. 2020; 93(1105): 20190551, doi: 10.1259/bjr.20190551, indexed in Pubmed: 31573326.
14. Garza Leal JG, Hernandez Leon I, Castillo Saenz L, et al. Laparoscopic ultrasound-guided radiofrequency volumetric thermal ablation of symptomatic uterine leiomyomas: feasibility study using the Halt 2000 Ablation System. J Minim Invasive Gynecol. 2011; 18(3): 364-371, doi: 10.1016/j.jmig.2011.02.006, indexed in Pubmed: 21545960.

15. Kim HS, Tsai J, Jacobs MA, et al. Percutaneous image-guided radiofrequency thermal ablation for large symptomatic uterine leiomyomata after uterine artery embolization: a feasibility and safety study. J Vasc Interv Radiol. 2007; 18(1 Pt 1): 41-48, doi: 10.1016/j.jvir.2006.10.010, indexed in Pubmed: 17296703.

16. Bergamini V, Ghezzi F, Cromi A, et al. Laparoscopic radiofrequency thermal ablation: a new approach to symptomatic uterine myomas. Am J Obstet Gynecol. 2005; 192(3): 768-773, doi: 10.1016/j.ajog.2004.10.591, indexed in Pubmed: 15746670.

17. Ghezzi F, Cromi A, Bergamini V, et al. Midterm outcome of radiofrequency thermal ablation for symptomatic uterine myomas. Surg Endosc. 2007; 21(11): 2081-2085, doi: 10.1007/s00464-007-9307-8, indexed in Pubmed: 17514400 .

18. Zhang J, Feng $L$, Zhang B, et al. Ultrasound-guided percutaneous microwave ablation for symptomatic uterine fibroid treatment-a clinical study. Int J Hyperthermia. 2011; 27(5): 510-516, doi: 10.3109/02656736.2011.562872, indexed in Pubmed: 21756048.

19. Chudnoff SG, Berman JM, Levine DJ, et al. Outpatient procedure for the treatment and relief of symptomatic uterine myomas. Obstet Gynecol. 2013; 121(5): 1075-1082, doi: 10.1097/AOG.0b013e31828b7962, indexed in Pubmed: 23635746.

20. Levens ED, Wesley R, Premkumar A, et al. Magnetic resonance imaging and transvaginal ultrasound for determining fibroid burden: implications for research and clinical care. Am J Obstet Gynecol. 2009; 200(5): 537.e1-537.e7, doi: 10.1016/j.ajog.2008.12.037, indexed in Pubmed: 19268886.

21. Turtulici G, Orlandi D, Dedone G, et al. Ultrasound-guided transvaginal radiofrequency ablation of uterine fibroids assisted by virtual needle tracking system: a preliminary study. Int J Hyperthermia. 2019; 35(1): 97-104, doi: 10.1080/02656736.2018.1479778, indexed in Pubmed: 30012030. 